\title{
Audiovisual Annotation in the Study of Physics
}

\author{
João Marçal $\dagger$ \\ Faculdade de Engenharia \\ Universidade do Porto \\ Porto Portugal \\ jonimar@gmail.com
}

\author{
Maria Manuel Borges \\ Faculdade de Letras, Coimbra \\ Universidade de Coimbra \\ Coimbra Portugal \\ mmb@fl.uc.pt \\ Paulo Carvalho \\ Faculdade de Ciências \\ Universidade do Porto \\ Porto Portugal \\ psimeao@fc.up.pt
}

\author{
Paula Viana \\ INESC-Tec and Polytechnic of Porto \\ Porto Portugal \\ paula.viana@inesctec.pt
}

\begin{abstract}
The support of video in the learning environment is nowadays used to many ends, either for demonstration, research or share. It is intended to reinforce the space before and after class and introduce a new dynamic and interaction in the classroom itself. Pedagogical innovation may be achieved by different approaches to motivate students and obtain better results. This paper presents a revision of the literature about the potential of using video annotation in the education context, specifically in the domain of Physics, using an open source annotation tool. The creation of audiovisual references, either for quick access to parts of organized video annotated content by the teacher, knowledge building or revision by and for other students is analyzed. This study is complemented with a testbed, showing the potential of using audiovisual annotated content, within a k-12 context. Students were invited to select video content, annotate, organize and publish the annotations, which could support the learning process in the domain of Physics. Results show that most of the aspects under analysis received a positive evaluation. The only exception relates to the capacity of the approach to motivated students to the study of Physics, as most of the students did not see this methodology as a motivating means.
\end{abstract}

\section{CCS CONCEPTS}

- Computer systems organization $\rightarrow$ Applied computing $\rightarrow$ Education $\rightarrow$ Interactive learning environments; • Information systems applications $\rightarrow$ Multimedia information systems;

\section{KEYWORDS}

Education; Active Learning; Physics; Video Annotation;

ACM Reference format:

João Marçal, Maria Manuel Borges Paula Viana and Paulo Carvalho. 2018. Audiovisual Annotation in the Study of Physics. In Proceedings of TEEM conference (TEEM'18). TEEM, Salamanca, Spain, 5 pages.

\section{RELATED WORK}

Since the work of Latour and Woolgar [1] on the central importance of inscriptions in the organization of scientific knowledge, the role of these parallel registers has become a major focus of research. Instead of reflecting spoken language, these external representations complement it by using the distinctive features of the material world to organize phenomena in a way that the spoken language does not allow. As technology developed, the use of video and the creation of annotation techniques helped researchers systematize their observations $[\underline{2}$, $3,4]$. These techniques involve the detailed recording of events on paper, specifying features such as durations, classifications / levels and temporal codes [ [5].

In Roth's and McGinn's [6] work, a new theoretical perspective on representations, derived from academic studies in science and technology, is approached around the notion of inscriptions and graphic representations, available through some support. The authors consider two types of writing tools: paper and pen, and computer. They claimed at the time that much was to be availed by the use of the computer. We can do the parallelism of the analogical inscription on paper, to the concept of digital annotation that can be used by the members of the community. In an educational context, it may refer to notes made by teachers or students. Marshall [7] defines annotation as "a tangible demonstration of student engagement with the text," investigating how readers interact with text, in which features such as readability, annotation, and navigation are examined as aspects that e-books have inherited from their print legacy. For Chen and Huang [ $\underline{8}$ ] it is "an active reading strategy where key information is written on the margins of our text, since it gives it a purpose, and the annotation helps to focus while reading, and really helps you learn from the text."

Digital annotations have clear advantages over paper annotations, as they can be searched, shared, archived and easily manipulated [9]. Video annotation has a spatial dimension, as in paper annotations, and a temporal dimension, i.e. the time interval in the video in which the annotation is associated. For Burr [10] the emergence of technology as an auxiliary tool in video annotation 
has increased reliability, repeatability and optimization of workflow.

Of the various studies carried out regarding the use of educational content besides the textual, specifically audiovisual, surges the concept of hypervideo (links within the video). In the study by Debevc, Šafarič, and Golob [11] a prototype was created and evaluated in order to verify if it provides sufficient information, so that students fully understand the subject. The structure of the hypervideo application consists of scenes and sequences of narration. A scene is defined as a set of frames displayed sequentially and relating to the same concept. For example, it could include the images showing first all the equipment and then the experiment showing the magnetic ball closest to the plane this allows observation of the movement of the ball in its own position. A scene can be shared by several narrative sequences, but the context of the scene may change in each. Narrative sequences represent a potential path or segment through a set of embedded video scenes and synchronized hypertext, sometimes dynamically mounted based on user interaction or in the context of scenes.

The analysis of users (both teachers and students) done by [11], identified the following requirements in the use of this type of tools:

- Applications should create a real learning environment with all interactive elements.

- Multimedia applications must be simple and understandable, and video-enabled.

- Systems need to include a navigation template.

- Systems need to include clear segments that users can choose.

As a reinforcement of academic practice through the use of video, Bartholomew [12] investigated the use of video as an enabling technology for teaching, for research, and for the involvement of stakeholders in curriculum design.

The collaborative knowledge building approach introduced by Scardamalia and Bereiter [13], based on the CSILE platform, was based on the belief that students represented a resource that was wasted and could be leveraged through network technology. CSILE, according to the authors, "restructured the flow of information in the classroom, so that questions, ideas, criticisms, suggestions, and other similar things were contributions to a public space equally accessible to all, instead of everything passed by the teacher or (as in e-mail) passing as messages between individual students. By linking these contributions, students created an emerging hypertext that represented collective knowledge, not just individual knowledge of the participants." [13]

In the literature we find reference to the difficulties in the teaching of Physics [14], namely in the transmission of basic concepts, as well as methodologies of correction in order to mitigate the difficulties. DiSessa [15] points out that one of the problems in the teaching of Physics stems from the fact that students do not feel themselves to be participants in the learning process. It also considers that the use of everyday examples, in the explanation of theoretical concepts, tends to involve students more in the discussion in the classroom, in order to improve their scientific understanding. The learning of physical processes on the basis of everyday objects is viewed by this author as an essential step to make processes acceptable and applicable to a wider range of circumstances. Redish [16] refers to the problem of students with different abilities memorizing without understanding, despite the most elaborate methodologies of exposition. Or students with reasonable ability to construct a graph, but not be able to understand its meaning. This author refers to several methods and resources that can be used in the teaching of Physics. One good example is the Just.in.Time Physics (JiTT) methodology, based on the interaction between didactic content on the Web and the tasks that are developed in the classroom or laboratory. [17]

In line with this approach one of the hypotheses to support the thesis in progress is that the provision of annotated contents should be able to improve the training process by promoting active learning using audiovisual annotations. This should allow students to practice processes that will have to be applied in the laboratory, or even consolidate concepts of the discipline under study in the improvement of the training process.

The JiTT methodology was initially developed to promote student's learning in the field of Physics by combining modified lectures, group discussion problem solving and Web technology. This approach focuses on two cognitive principles involving students and teachers in the teaching / learning process: the first one states that students learn more effectively if they are intellectually involved; the second mentions that teachers teach better if they understand what their students think and know [17]. The implementation of this approach depends, according to the authors, on 3 factors: a mechanism for delivering questions through the Web and for collecting and visualizing the answers in an appropriate way; a series of exercises designed to allow students to easily understand the contents; and a teacher who can understand the difficulties of the students in order to lead the discussion in the classroom.

Inspired by the study of Novak [17], Simkins and Maier [18] introduced JiTT into self-taught economics courses, requiring students to answer questions related to the material in the next class, a few hours before class using a course management system online. The results were positive and immediate: students came to class better prepared and reported that JiTT exercises helped to focus and organize their studies outside of class. In addition, students' responses to JiTT questions highlighted gaps in their learning, visible to teachers before class. This knowledge allowed teachers to create classroom activities that directly addressed these learning gaps while the material was still fresh in students' minds - hence the expression "just-in-time" - leading to improved learning. For the authors, the JiTT approach suggests that the classroom is not an assembly line, but rather a learning environment that needs to be adjusted to what students know and bring into the classroom.

The Active Learning methodology taken in the sense given by Knight [19] and Hake [20], that is, as a teaching philosophy where students construct their own knowledge, through interaction with materials and ideas, instead of passive participants in the 
knowledge process. The implementation of strategies according to this methodology has the following characteristics:

- Students spend most of the class actively involved in doing / thinking / speaking Physics - and not listening to someone talk about it.

- Students interact with their peers.

- Students receive immediate feedback from their work.

- The instructor is more a facilitator, less a transmitter of knowledge.

- Students are responsible for their knowledge. This includes engaging in activities, text study, and performing tasks.

Allen and Tanner [21] defined active learning as "seeking new information, organizing it meaningfully and having the opportunity to explain it to others". Researches have shown that the use of such active learning methods improves students' learning and their attitudes towards studying [르, 23, 24].

\section{INTRODUCTION}

This research is based on the hypothesis that video annotations can contribute to the teaching / learning process in the area of Physics. Based on the CLIPPER prototype audiovisual annotation tool, the main objective will be to realize the importance that the annotation of video contents plays in the improvement of the educational process.

In relation to the study research questions, they are the following:

- $\quad$ Can students feel more motivated in their study using video annotations?

- Does the use of video annotations assist the teacher in the interpretation of concepts and physical phenomena by students?

The first question seeks to verify if this type of study method can help the traditional study by the students. The second question is intended to be comparative-causal, to investigate the relationship between students' motivation and their performance in their study.

The experiment presented in this paper partially answers the first two research question and provides good insights on the use of the described approach.

\section{TESTBED}

\subsection{Annotation Tool}

CLIPPER [25] is a free web-based tool that allows/permits users to create a catalog of video annotations and share individual annotated clips or clips that are within a specific list of clips.

This tool allows the user to specify a section of the video, hereinafter referred to as a segment or clip, directly from the player's timeline of the video imported by the user, identified through the video URL (for example, a YouTube URL). These segments, identified by the initial and final playback instant, are anchored so that the user can view the clips identified by the user instead of having to view the entire video. On the other hand, the user can insert text annotations and keywords that accompany each of the user-created segments.
Once the application provides all the functionalities required for the intended experiment, it was selected as the tool to be used within this testbed.

\subsection{Application Scenario}

The testbed was fully implemented in a class of $20 \mathrm{k}-10$ students who selected the videos to be annotated, used CLIPPER to annotate selected videos and built a website for publicizing the annotated content.

In the first stage of the project, the students reviewed the curricular goals of Physics and Chemistry A curricular program, specifically concerning the contents of Physics. A list of all the contents of the curriculum that could be complemented by annotated videos to assist their study was built. The students recalled the organization of the k-10 Physics curricular by domains and subdomains: the first domain, titled "Energy and movements", has 8 subdomains; the second, entitled "Energy and electrical phenomena", is composed of 7 subdomains; and the third, "Energy and thermal phenomena and radiation", is composed of 10 subdomains. This organization of the contents was always followed during the project to facilitate the attribution of the selected videos to the contents of the program.

A web search for videos of relevant content that may prove useful for studying the concepts of physics in each domain and subdomain was done. This research was performed by the students involved in the project, who recorded almost 200 videos. After a more detailed analysis and later validation by the teachers, 95 videos were identified to be annotated with the CLIPPER tool, as they are potentially useful in the study of several concepts of the different topics.

The video annotation stage was divided into three phases:

1) tutorial session for the students.

2) student familiarization with the tool through a hands-on

session with example videos.

3) annotation of the 95 videos.

In the introduction session to annotate the videos, the students were assisted in using the tool to create annotations. At this stage, it was exemplified what is meant to be an annotation and some exercises of annotations were done in illustrative videos in a large group.

On the second step, the students performed several annotation tasks and some doubts were overcome.

In the third phase, the students annotated the 95 videos, segmenting them into clips that point to the most relevant content and added a summary and some illustrative keywords. The annotation review was done in pairs.

To enable a broader disclosure of the work done, so that it could be of benefit to a larger group of students, a website was created [26] and promoted within the group of students that will begin the k-10 and those who are attending the k-11, so they can use the contents to review some concepts.

This process was documented in video [27] and shared between the CLIPPER developer team and community. 


\subsection{Evaluation Process}

The evaluation process included two aspects:

1) The teacher in charge analyzed the output of the annotation process and classified each group according to a set of pre-defined parameters;

2) Each student answered a survey.

The teacher's evaluation focused on four aspects of the learning process:

1. Clarity in the presentation (organization of video clips) [10\%]

2. Creativity / originality (what kind of videos was chosen) [40\%]

3. Subject agreement (if the clip was in accordance with the topic) [10\%]

4. Content (annotation quality) [40\%]

To measure the students' perception about the annotation process, a 4 point Likert scale was used, to evaluate six statements related to the annotation experience. The objectives were:

1. To assess the pertinence of this type of study methodology.

2. To verify if this form of study motivated the student

3. To understand the usefulness of annotations in the understanding of themes.

4. To ascertain whether the fact of video annotation being carried out in a group is an advantage or disadvantage.

5. Based on the possibility of the annotation platform making it possible to work with the same account by several users, it is intended to assess the advantage of this functionality in the context of group work

6. To determine if the group notation facilitates the mutual aid in the annotation

To get additional data, three open questions were asked:

a) What aspects did you dislike in this annotation work?

b) What aspects did you like in the annotation work?

c) Comments or ideas to help improve the application? This open questions served as feedback to troubleshooting and improvement either in the teaching methodology or the technology itself. This information proved to be valuable for the developers of CLIPPER, in detecting bugs unnoticed within learning context use.

\section{RESULTS}

Some conclusions can be highlighted from the analysis of the results: most of the aspects under analysis received a positive evaluation. The only exception relates to the capacity of the approach to motivated students to the study of Physics as most of the students did not see this methodology as a motivating means. One factor that may have contributed to this result is the fact that, because it was their first hands-on experience with the clipper platform, these students faced some problems in its use. Tasks such as sorting the videos in the "playlist mode", pausing some videos when playing and cropping the time were not easily implemented. This resulted in extra time devoted to a working process which might have influenced their perceived benefits.
On the other hand, all the other aspects revealed positive: most students agreed on the contribution that video annotations made to understanding concepts in physics; the collaborative annotation strategy also seems to have contributed to a better understanding of the students' doubts, that individually or on writing could be more difficult to clarify and to make the process easier; functionalities provided by the CLIPPER tool to organize content (the clip lists) also proved to be useful.

Table 1 presents the collected results to the 6 scaled questions of the 20 students' pool. The weighted mean and standard deviation values are also shown. The weight of the questions 1 and 2 have $20 \%$ each, and questions from 3 to 6 have $15 \%$ each.

Table 1. Results from survey applied to the students (1. Totally disagree...4 Totally agree)

\begin{tabular}{|c|c|c|c|c|c|c|}
\cline { 2 - 6 } \multicolumn{1}{c|}{} & \multicolumn{9}{c|}{ Scale } & \multicolumn{1}{c|}{} \\
\hline Question & $\mathbf{1}$ & $\mathbf{2}$ & $\mathbf{3}$ & $\mathbf{4}$ & Mean & SD \\
\hline $1^{*}$ & 1 & 5 & 14 & 0 & 2,65 & 0,57 \\
\hline $2^{*}$ & 3 & 12 & 5 & 0 & 2,1 & 0,62 \\
\hline $3^{*}$ & 0 & 4 & 16 & 0 & 2,8 & 0,4 \\
\hline $4^{*}$ & 1 & 4 & 15 & 0 & 2,7 & 0,55 \\
\hline $5^{*}$ & 1 & 5 & 11 & 3 & 2,8 & 0,74 \\
\hline $6^{*}$ & 1 & 6 & 13 & 0 & 2,6 & 0,58 \\
\hline
\end{tabular}

The questions were as following:

$1^{*}$ Taking notes in the videos helped me understand concepts in physics.

$2^{*} \quad$ When taking notes, I became more motivated to study physics.

$3^{*}$ The video annotations provided by colleagues allowed a better understanding of the concepts.

$4^{*}$ The collaborative process of video annotation has contributed to a quicker understanding of content.

$5^{*}$ Creating the clip list contributed to improving the collaborative annotation process.

$6^{*}$ Group annotation work make the annotation process easier.

The open questions also gave some clues on aspects that could be improved either in the tool or in the process. Although the tool was considered adequate for the annotation purpose, a set of functionalities to be included in a future upgraded version were identified. The most relevant are: annotations could be more intuitively perceived if they were overlapping the video content instead of being presented in a separate area; navigation functionalities within the annotations should be improved. The open questions also contributed to strength the conclusions from the scaled questions as students highlighted the ability of the approach to help consolidating the subject and helping to acquire knowledge. They also mentioned this could be an interesting complementary study method. Students overall expressed that the main benefit of this time-based annotations was to enable then to optimize their study by watching summarized versions of the content instead of reading and watching long videos. 


\section{CONCLUSIONS}

One of the overall goals of this research is to understand whether students can feel more engaged in the study of subjects in which they usually face difficulties and if an innovative approach could enable better scholar results. For that, we argue that, given that current high school students are quite keen to using audiovisual content, enriching their standard study methods through the use of audiovisual content manipulation might be a solution.

This first testbed allowed to test the CLIPPER tool and refine the application methodology to future students and teachers.

Overall the results were positive, considering that this was the first time this methodology was tested and adjustments and improvements will be needed.

There may be several ways of using the video annotation tool that may also be exploited. This will depend on the teaching strategy of each teacher to prepare students for the assessments or, for example, in the construction of a "recorded bookmarked video library" that can be used by other students at the beginning of a curricular topic.

Future work includes making similar experiments in a larger pool of students and focusing on the teacher's use of video annotations to support his teaching.

\section{ACKNOWLEDGMENTS}

I would like to thank Professor Nuno Moutinho for the opportunity to testbed Clipper at EscolaGlobal, and Professor José Pinto in the bridge with the students. Also John Casey, responsible for the CLIPPER tool, for collaborating in this study.

\section{REFERENCES}

[1] Bruno Latour and Steve Woolgar. 1979. Laboratory Life. Beverly Hills.

[2] Laura L Lee. 1974. Developmental sentence analysis: A grammatical assessment procedure for speech and language clinicians. Northwestern University Press.

[3] Kristine S Retherford. 2000. Guide to analysis of language transcripts. Thinking Publications.

[4] Katherine L Rosenblum, Charles Zeanah, Susan Mcdonough, and Maria Muzik. 2004. Video-taped coding of working model of the child interviews: a viable and useful alternative to verbatim transcripts? Infant Behavior and $\begin{array}{llll}\text { Development } & 27, & 4, & 544-549 .\end{array}$ http://dx.doi.org/10.1016/j.infbeh.2004.04.001.

[5] Barbara J Leadholm and Jon F Miller. 1994. Language Sample Analysis: The Wisconsin Guide. Bulletin 92424.

[6] Wolff-Michael Roth and Michelle K Mcginn. 1998. Inscriptions: Toward a theory of representing as social practice. Review of educational research 68 , 1, 35-59. DOI: http://dx.doi.org/10.3102/00346543068001035.

[7] Catherine C Marshall. 2009. Reading and writing the electronic book. Synthesis lectures on information concepts, retrieval, and services 1, 1, 1-185. DOI: http://dx.doi.org/10.2200/S00215ED1V01Y200907ICR009.

[8] Chih-Ming Chen and Sheng-Hui Huang. 2014. Web-based reading annotation system with an attention-based self-regulated learning mechanism for promoting reading performance. British fournal of Educational Technology 45, 5, 959-980. DOI: http://dx.doi.org/10.1111/bjet.12119.

[9] Michael Shilman and Zile Wei. 2004. Recognizing freeform digital ink annotations. In International Workshop on Document Analysis Systems. Springer 322-331. DOI: http://dx.doi.org/10.1007/978-3-540-28640-0 30.

[10] Brandon Burr. 2006. VACA: a tool for qualitative video analysis. In $\mathrm{CHI}^{\prime} 06$ extended abstracts on Human factors in computing systems ACM 622-627. DOI: http://dx.doi.org/10.1145/1125451.1125580.

[11] Matjaž Debevc, Riko Šafarič, and Marjan Golob. 2008. Hypervideo application on an experimental control system as an approach to education. Computer Applications in Engineering Education 16, 1, 31-44. DOI: http://dx.doi.org/10.1002/cae.20116.
[12] Paul Bartholomew. 2014. Enhancing academic practice through the use of video: a longitudinal case study of professional development in higher education. In School of Education University of Birmingham, 324.

[13] Marlene Scardamalia and Carl Bereiter. 2005. Knowledge Building. In The Cambridge Handbook of the Learning Sciences, R.K. Sawyer Ed. Cambridge University Press, Cambridge, 97-115. DOI: http://dx.doi.org/10.1017/CBO9780511816833.008.

[14] A. B. Arons. 1997. Teaching introductory physics. Wiley, New York.

[15] Andrea A Disessa. 1993. Toward an epistemology of physics. Cognition and $\begin{array}{llll}\text { instruction } & 10, & 2-3, & 105-225 .\end{array}$ http://dx.doi.org/10.1080/07370008.1985.9649008.

[16] Edward F. Redish. 2003. Teaching physics with the physics suite. John Wiley \& Sons Hoboken, NJ.

[17] Gm Novak, A Gavrini, W Christian, and E Patterson. 1999. Just-in-time teaching: Blending active learning with web technology. . Upper Saddle River NJ: Prentice Hall.

[18] Scott Simkins and Mark Maier. 2010. Just-in-time teaching: Across the disciplines, across the academy. Stylus Publishing, LLC.

[19] Randall Dewey Knight. 2004. Five easy lessons : strategies for successful physics teaching. Addison Wesley, San Francisco, Calif.

[20] Richard R Hake. 1998. Interactive-engagement versus traditional methods: A six-thousand-student survey of mechanics test data for introductory physics courses. American Journal of Physics 66, 1, 64-74. DOI: http://dx.doi.org/10.1119/1.18809.

[21] Deborah Allen and Kimberly Tanner. 2005. Infusing active learning into the large-enrollment biology class: seven strategies, from the simple to complex. Cell Biology Education 4, 4, 262-268. DOI: http://dx.doi.org/10.1187/cbe.05-08-0113.

[22] Lev S Vygotsky. 1978. Mind in society: The development of higher mental process. Cambridge, MA: Harvard University Press.

[23] Arthur W Chickering and Zelda F Gamson. 1987. Seven principles for good practice in undergraduate education. AAHE bulletin 3, 7 .

[24] Peter Armbruster, Maya Patel, Erika Johnson, and Martha Weiss. 2009. Active learning and student-centered pedagogy improve student attitudes and performance in introductory biology. CBE-Life Sciences Education 8, 3, 203-213. DOI: http://dx.doi.org/10.1187/cbe.09-03-0025.

[25] Clipper. Retrieved from https://clipperdev.com/ .

[26] Aprender Física com o Clipper. 2018. Retrieved from https://clipperfisica.wordpress.com/ .

[27] Clipper in Portugal. 2018. Retrieved from http://blog.clippertube.com/index.php/2018/06/07/clipper-in-portugal/ . 